\title{
Quantitative analysis of electric force microscopy: The role of sample geometry
}

\author{
Emma Tevaarwerk, D. G. Keppel, P. Rugheimer, M. G. Lagally, and M. A. Eriksson ${ }^{\text {a) }}$ \\ University of Wisconsin-Madison, 1150 University Avenue, Madison, Wisconsin 53706
}

(Received 28 October 2004; accepted 3 March 2005; published online 26 April 2005)

Quantitative electric force microscopy (EFM) is usually restricted to flat samples, because vertical sample topography traditionally makes quantitative interpretation of EFM data difficult. Many important samples, including self-assembled nanostructures, possess interesting nanoscale electrical properties in addition to complex topography. Here we present techniques for analysis of EFM images of such samples, using voltage modulated EFM augmented by three-dimensional simulations. We demonstrate the effectiveness of these techniques in analyzing EFM images of self-assembled SiGe nanostructures on insulator, report measured dielectric properties, and discuss the limitations sample topography places on quantitative measurement. (C) 2005 American Institute of Physics. [DOI: 10.1063/1.1898183]

\section{INTRODUCTION}

The electrostatic forces on a conducting cantilever depend strongly on the nanoscale electrical properties of the sample beneath it. This electrostatic interaction is used in electric force microscopy (EFM) to quantitatively measure the local charge, dielectric constant, and work function of relatively flat samples. ${ }^{1-6}$ If the sample is not flat, EFM measurements are notoriously difficult to interpret, primarily because topography strongly influences tip-sample electrostatics. Consequently, attributing EFM signals to either topographic or electronic origins can be challenging. Because many interesting nanocircuits and self-assembled nanostructures possess significant topography, it is important to develop techniques that can overcome these challenges and extract information about local dielectric constant, trapped charge, and work function.

One effective way to distinguish electrical properties from topographic crosstalk is to exploit time-varying phenomena. Transient responses such as charge decay, blinking, or moving under the influence of an applied voltage have been used to quantitatively measure local trapped charge. ${ }^{2,3,5,7}$ Unfortunately, measurement of static properties such as local dielectric constant precludes the use of these approaches. Tip-sample simulations of EFM (Refs. 9-14) have been shown to be helpful in the extraction of physical quantities from EFM images of essentially flat samples. However, simulations may in fact be most useful for samples with three-dimensional nanostructured topography.

We present techniques that allow the effect of sample geometry on the interpretation of EFM images to be systematically examined and understood. These techniques utilize voltage-modulated EFM (VMEFM) augmented by threedimensional electrostatic simulations. We provide guidelines to indicate when simulations which take sample topography

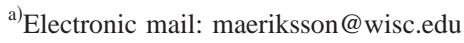

into account are necessary to interpret EFM images. We discuss the effect of sample topography in the context of selfassembled SiGe nanostructures, and we match experimentally measured EFM data with simulated scanlines to extract dielectric properties. We show that carefully calibrated cantilever force constants are not required for quantitative analysis, because simple electrostatic references are frequently present in, or can be easily added to, samples of interest.

\section{EXPERIMENTAL AND COMPUTATIONAL APPROACH}

In VMEFM a conducting cantilever oscillates at a set height above the surface of the sample and probes the longrange electrostatic forces between tip and sample. At any given point over the sample, the resonance frequency of the cantilever is shifted an amount $\Delta v$ by the local force gradient. For small amplitudes of cantilever oscillation, this shift is given by

$$
\Delta v=-\frac{v_{o}}{2 k} F^{\prime},
$$

where $k$ is the cantilever stiffness, $v_{o}$ is its natural resonance frequency, and $F^{\prime}$ is the local tip-sample force gradient. ${ }^{15,16}$ In general, the measured frequency shift is difficult to simulate accurately because the force on the cantilever, and thus the EFM response, depends on many variables, such as charge, workfunction, dielectric constant, and geometry.

In VMEFM, the application of a voltage $V_{\mathrm{tip}}=V_{\mathrm{dc}}$ $+V_{\text {ac }} \sin (\omega t)$ simplifies data analysis. The ac voltage produces a frequency shift $\Delta v$ with Fourier components at dc, $\omega$, and $2 \omega$. We focus on the response at $2 \omega{ }^{1}$

$$
\Delta v_{2 \omega}=-\frac{v_{o}}{2 k} F_{2 \omega}^{\prime}=\frac{v_{o}}{2 k}\left(\frac{V_{a c}^{2}}{4}\right)\left(\frac{\partial^{2} C}{\partial z^{2}}\right) \cos (2 \omega t),
$$

because it depends only on the second derivative of the tipsample capacitance $C^{\prime \prime}(z)$, the applied ac voltage $V_{\text {ac }}$, the cantilever stiffness $k$, and the natural resonance frequency $v_{o}$. 


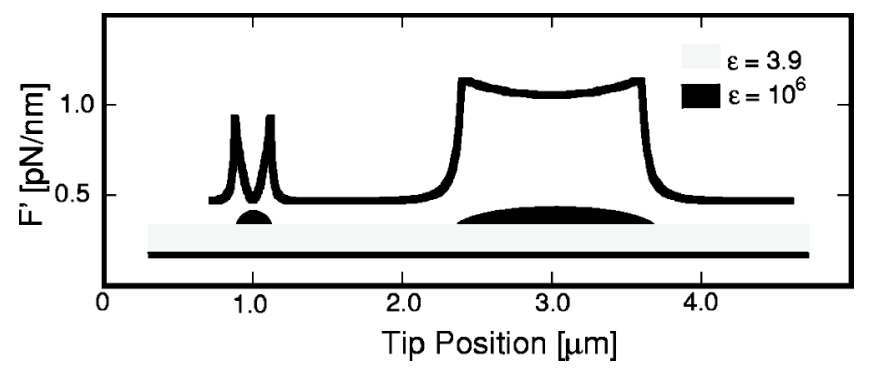

FIG. 1. Simulated EFM scanline of a tip it scans at a set height of $20 \mathrm{~nm}$ over floating nanostructures with the same dielectric constant but different topographies. The nanostructure on the left (diameter $100 \mathrm{~nm}$ and height 40 $\mathrm{nm}$ ) has strong edge peaks and little increase at its center, while the larger nanostructure (diameter $600 \mathrm{~nm}$ and height $40 \mathrm{~nm}$ ) on the right has small edge peaks and a large increase at its center.

Thus, $\Delta v_{2 \omega}$ is a measure of anything that influences the tipsample capacitance, such as the local dielectric constant and geometry. Although this type of VMEFM ( $2 \omega$-EFM) has received relatively little attention, ${ }^{17-20}$ it is ideal for investigating the interplay between geometry and local dielectric constant. Because $2 \omega$-EFM does not require electrical contact to the sample surface-only a backgate is required-it is also particularly useful for samples with electrically isolated or floating elements, such as nanowires ${ }^{21}$ or nanotubes ${ }^{22}$ on oxide.

As shown in the simulations in Fig. 1, described below, for nanostructures with significant vertical topography, the lateral size of a nanostructure can be very important in determining the EFM response. Although the two nanostructures in Fig. 1 have identical dielectric constants, they produce radically different EFM responses. For the simulated scan over the larger nanostructure, an increased force gradient over its center is present. In contrast, the scan over the smaller nanostructure is low in the middle and has two sharp peaks at its edges, which result primarily from the geometry of the nanostructure. These two circumstances occur routinely in EFM scans of nanostructured materials with vertical topography. Without knowledge of how sample geometry influences measured EFM responses, it can be difficult to know whether EFM images are a good measure of local dielectric properties.

To create the scanlines, we solve Laplace's equation in three dimensions at several tip-sample separations using FLEXPDE. ${ }^{23,24}$ We find the force on the cantilever at several heights, curve fit the resulting forces with a power law, ${ }^{25}$ and differentiate to obtain the force gradient on the tip. In the simulations the tip has the dimensions of the commercial cantilever [(MESP), Veeco Metrology, Santa Barabara, CA] and consists of a pyramid with front and back edges at 10 deg and $25 \mathrm{deg}$ and side edges at $17 \mathrm{deg}$ from vertical. A paraboloid is superimposed at the apex of the tip to provide a tip radius of curvature of $25 \mathrm{~nm}$. A flat plate is added at the top of the pyramid to simulate the cantilever arm.

Figure 1 implies that there is some lateral length scale which, when compared to nanostructure size, determines the scanline shape. The lateral size of the nanostructure is important because it determines how much the nanostructure perturbs the electric field beneath the tip. This in turn depends on how the electric field lines are concentrated be- (a)

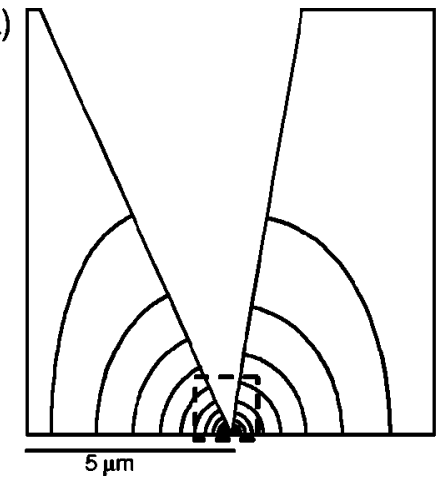

(b)

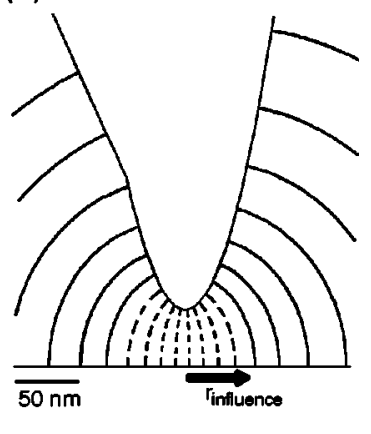

FIG. 2. Calculated electric field lines for: (a) full tip $50 \mathrm{~nm}$ from a conducting plate, and (b) apex of the same tip, showing the field lines (dashed) contributing $50 \%$ of the total force gradient.

tween the tip and sample. As shown in Fig. 2(a), because of the shape of the tip, the majority of the electric field lines between a cantilever and a flat metal sample are concentrated directly beneath the tip. In fact, EFM has high resolution because the forces important for establishing the tip-sample force gradient are found predominantly at the apex of the tip. We can quantify the importance of each region of the tip by calculating the force gradient on larger and larger fractions of the tip beginning at the apex. We find those responsible for $50 \%$ of the full sample-cantilever interaction, depicted as dashed lines in Fig. 2. ${ }^{12}$ By following the electric field lines from the appropriate portions of the tip down the conducting sample, we find the area of the sample responsible for $50 \%$ of the force gradient. We describe this area of the sample by its radius, which we call the radius of influence. The size of $r_{\text {influence }}$ depends largely on the distance $z$ between the tip and the nearest conducting layer in the sample. Using threedimensional (3D) simulations, we can quantify $r_{\text {influence }}$ for MESP cantilevers at a height $z$ (typically $>10 \mathrm{~nm}$ ) above a flat conducting plate: For techniques that measure local force gradient $r_{\text {influence }}=0.8 z+4 \mathrm{~nm}$, while $r_{\text {influence }}=3 z-5 \mathrm{~nm}$ for techniques that measure the local force. ${ }^{26}$ If a dielectric of thickness $z^{\prime}$ separates the tip and the nearest conducting plate, the radius of influence can still be estimated by adjusting the tip height to account for the dielectric, $z$ $\approx z^{\prime} / \varepsilon_{\text {dielectric }}$. These equations demonstrate explicitly the fact that measuring force gradient rather than force increases EFM resolution. ${ }^{9}$

In the context of the radius of influence, we systematically investigate the importance of the lateral size of a nanostructure. Figure 3 plots the force gradient on a tip over conducting nanostructures of varying radius $r_{\mathrm{ns}}$ and a height of $40 \mathrm{~nm}$. For "small" nanostructures $\left(r_{\mathrm{ns}} \ll r_{\text {influence }}\right)$, the presence of the nanostructure beneath the tip actually reduces the force gradient from what it would be without any nanostructure at all (dashed line). This is simply because the tip is farther away from the sample and the dielectric nature of the nanostructure has little impact. As the radius of the nanostructure increases, however, the nanostructure begins to influence many more field lines between the tip and the backgate. More of the electric field lines end on nanostructure itself, increasing the bending of the electric field lines and thus the local force gradient. For an infinitely large 


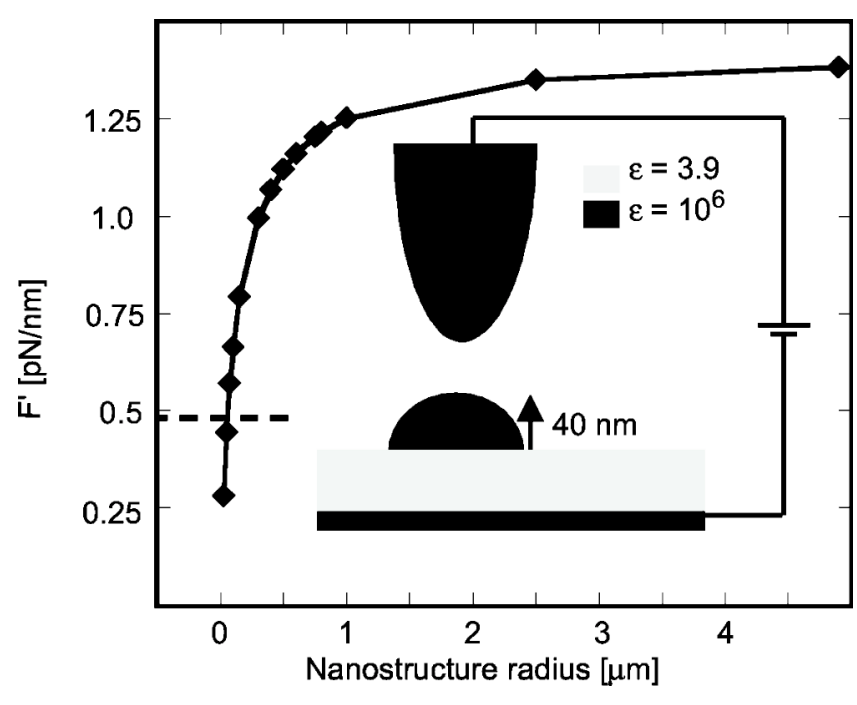

FIG. 3. Force gradient on stationary cantilever $20 \mathrm{~nm}$ above the center of a floating nanostructure with varying radius. For small nanostructure radii, the force gradient approaches the value of a tip $60 \mathrm{~nm}$ from the oxide; for large radii, the force gradient approaches that of a tip $20 \mathrm{~nm}$ from a flat conducting plate.

"nanostructure," the force gradient approaches a constant value because the sample is locally flat. We can define three regimes for nanostructures of this height: (1) $r_{\mathrm{ns}} \ll r_{\text {influence }}$, (2) $r_{\text {influence }} \sim r_{\mathrm{ns}}$, and (3) $r_{\mathrm{ns}} \gg r_{\text {influence. }}$. For $r_{\text {influence }} \sim r_{\mathrm{ns}}$ simulations which take into account nanostructure geometry are pivotal to data interpretation because the lateral size of the nanostructure directly affects the magnitude of the EFM response. For example, recent measurement of the local dielectric constant PAn.HCSA/PEO nanofibers by scanning capacitance microscopy showed that quantitative agreement between simulations and experiment could not be reached by modeling the sample as a flat plane. They subsequently conclude that the exact geometry of the nanofiber would need to be taken into account to quantitatively explain measurements. ${ }^{21}$ Using the parameters for this experiment in our equations, we predict $r_{\text {influence }} \sim 80 \mathrm{~nm}$; given that $r_{\mathrm{ns}}$ $\sim 80-150 \mathrm{~nm}$ one would expect that nanostructure geometry would indeed need to be taken into account for quantitative measurements. We note that for $r_{\mathrm{ns}} \ll r_{\text {influence }}$ and small nanostructure height, the sample is essentially flat and conventional simulations which do not account for sample geometry are often sufficient. Similarly, for $r_{\mathrm{ns}} \gg r_{\text {influence }}$ simulations which account for nanostructure geometry are less critical.

\section{DATA ANALYSIS}

We now turn to experimental EFM images in order to demonstrate quantitative data interpretation of a nanostructured sample with significant vertical topography. The sample consists of self-assembled SiGe nanostructures on silicon-on-insulator. Sample morphology and growth specifics are described in detail elsewhere. ${ }^{20}$ Briefly, a thin layer of silicon $(10 \mathrm{~nm})$ sitting on oxide is laterally patterned into $10 \mu \mathrm{m} \times 10 \mu \mathrm{m}$ mesas; $\mathrm{Ge}$ is then deposited at $700{ }^{\circ} \mathrm{C}$. The germanium diffuses to the edge of the mesa, where it forms (a)

(b)

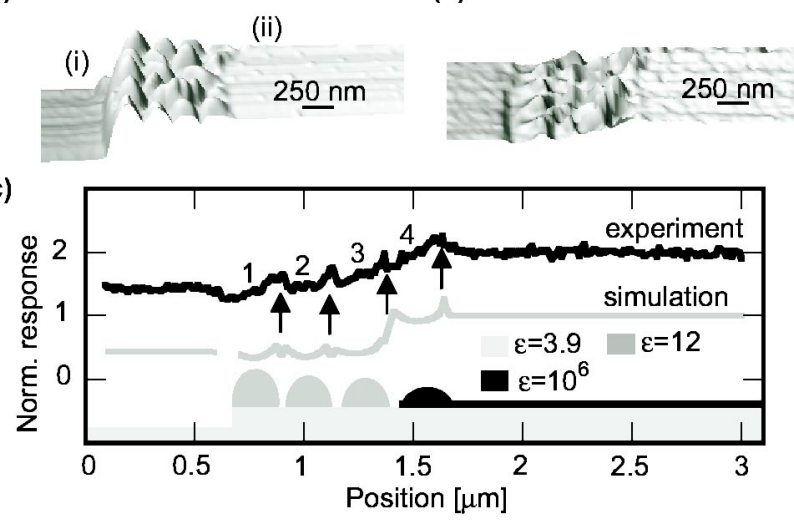

FIG. 4. Tapping mode atomic force microscopy (a) and $2 \omega$-electric force microscopy with $V_{\text {tip }}=2 \mathrm{~V}$ peak to peak at a frequency of $300 \mathrm{~Hz}$ and tip-sample separation $20 \mathrm{~nm}$ (b) of SiGe nanostructures at the edge of a mesa. (c) Normalized simulated scanline offset from normalized experimental scanline. The inset (not to scale) shows the predicted dielectric constants of the SiGe nanostructures.

SiGe domes. As the SiGe domes form, they incorporate the surrounding ultra-thin silicon layer, electrically isolating themselves from their surroundings. ${ }^{20}$

Figures 4(a) and 4(b) show tapping mode atomic force microscopy (AFM) and $2 \omega$-EFM images of the edge of such a mesa. Because of the complex sample topography, our EFM images are acquired in "lift-mode" on a Digital Instruments Nanoscope IIIa with an Extender Electronics Module. In this mode the cantilever makes one pass in tapping mode to acquire sample topography and then retraces the same scanline, using the acquired topographic data to hover over the sample at some set tip-sample separation. In the EFM measurements, the tip is electrically connected to a silicon handle wafer (backgate) underneath the nanostructures on oxide, similar to the situation shown in Fig. 3 (inset). The tip traces the topography at a height of $20 \mathrm{~nm}$, with an ac voltage of $2 \mathrm{~V}$ peak-to-peak and a frequency of $300 \mathrm{~Hz}$ applied. The measured frequency shift is fed into a lock-in amplifier and the response at $600 \mathrm{~Hz}$ is extracted. Because the tipsample separation is $20 \mathrm{~nm}$ and the oxide is $200 \mathrm{~nm}$ thick, $r_{\text {influence }}$ is a maximum of $\sim 60 \mathrm{~nm}$ (Ref. 27) over the domes. The radius of the nanostructures is $\sim 125 \mathrm{~nm}$, so $r_{\text {influence }}$ $\sim r_{\mathrm{ns}}$ and we would expect that 3D simulations that account for nanostructure geometry are necessary to extract dielectric information.

Before interpreting the more complicated EFM response over the SiGe nanostructures, we verify that simulations and experiments match well for simple geometries. Although simulated force gradients can be equated with measured frequency shifts using Eq. (2) (both give values in the tens of hertz), the accuracy of this prediction is limited by knowledge of cantilever spring constant. Instead, we choose two relatively simple electrostatic situations - the tip over a flat metal plate and the tip over an oxide-and compare the predicted ratio of these two electrostatic situations to the corresponding experimentally measured ratio. We use the ratio of the expected values for (i) a tip $20 \mathrm{~nm}$ from an insulator and (ii) a tip $20 \mathrm{~nm}$ from a conducting plate, similar to the experimental situations to the far left and right in Fig. 4(a). As 
we have shown previously, the thin layer of silicon on the mesa in situation (ii) has large enough conductivity that we may treat it as a conducting plane. ${ }^{20}$ Off the mesa [situation (i)] the silicon has been removed, exposing the silicon dioxide underneath. The predicted ratio of 2.5:1 for these two situations matches well with the experimentally measured ratio of 2.4:1, with no adjustable parameters.

In contrast to the response over the flat areas of the sample, the EFM response over the SiGe domes is quite complex. Figure 4(c) shows the predicted (bottom) and experimental (top) scanlines. For ease of comparison, both scanlines have been normalized with respect to the response on the mesa. For the experimental scanline, the SiGe domes are labeled 1-4, and the edge peaks between them are highlighted by arrows. The EFM responses over the domes one and two are relatively low (similar to the response off the mesa), while the response over the fourth domes is higher, similar to the response on the mesa. Because of the complex geometry of the SiGe nanostructures, this difference in response could result from (1) scanning over an edge, (2) the shape of the domes themselves, or (3) a variation in the local dielectric constant. It is the role of three dimensional simulations to differentiate between these possibilities.

We can account for possibilities (1) and (2) by inputting the exact geometry of the domes obtained from the tapping mode AFM scans into the electrostatic simulations. We limit the possible dome dielectric constants to $3.9\left(\mathrm{SiO}_{2}\right), 12-16$ ( $\mathrm{Si}$ to $\mathrm{Ge}$ ), and infinity (a good conductor, approximated here as $10^{6}$ ). From the AFM image, the silicon layer ends beneath the fourth dome from the edge. By holding simulation geometry constant and changing only the dome dielectric constant we produce several possible scanlines. We show here the only simulated scanline which best matches our experimentally measured scanline. The simulated scanline has a low response over domes one and two and a high response over dome four. Note also that the simulated scanline has edge peaks of appropriate magnitude in between each of the domes. This scanline predicts that the three edge domes have a dielectric constant of 12 (gray), while the fourth dome (black) has a dielectric constant of infinity $\left(10^{6}\right)$.

We would generally expect all of the SiGe nanostructures to have dielectric constants of the same magnitude, and thus the extracted result for dome four is somewhat surprising. To understand this result, recall that the silicon plate behaves as a conductor in these EFM experiments because it contains mobile charge. The innermost $\mathrm{SiGe}$ nanostructure is electrically connected to the silicon plate and therefore charge can flow freely from the plate into the nanostructure. As a result, the nanostructure can locally screen the tip from the backgate. The other three nanostructures are electrically isolated from the plate, and thus cannot draw free charge from it. They have a dielectric constant of order 12 because they possess insufficient free charge to screen the tip from the backgate.

We note that even with careful accounting for nanostructure geometry, the simulated and experimental scanlines do not perfectly match. In particular, the slope of the frequency shift over the third dome from the edge does not match exactly. This is probably because the silicon plate does not end at the exact lateral position we have assumed in our simulations - unfortunately this is one aspect of the sample topography we cannot measure precisely with tapping mode AFM. Although the simulations we report here enable significantly improved interpretation of the EFM images of the SiGe nanostructures on silicon-on-insulator, we are not able to distinguish between $\varepsilon=12(\mathrm{Si})$ and $16(\mathrm{Ge})$; however, we can easily distinguish between $\varepsilon=3.9\left(\mathrm{SiO}_{2}\right)$ and 12 (or 16) even in the presence of very large topography.

As we have shown, sample topography plays an important role determining the EFM response for nanostructured samples. We introduce the concept of the radius of influence and show that when sample features are on the order of $r_{\text {influence}}$, electrostatic simulations which accurately account for sample topography are needed to extract dielectric properties. As an example, we discuss the role that geometry plays in EFM measurements SiGe nanostructures on siliconon-insulator, and extract nanostructure dielectric properties. We show that use of simple electrostatic references can eliminate the need for carefully calibrated cantilever spring constants. Although we focus exclusively on $2 \omega$-EFM, analogous results should hold for other scanning probe microscopies (such as scanning conductance microscopy) in which geometry influences the measured response.

\section{ACKNOWLEDGMENTS}

This material is based upon work supported by the DOE under Grant No. DE-FG02-03ER46028, by the NSF under Grant No. DMR-0094063, DMR-0079983 and by the Research Corporation.

${ }^{1}$ B. D. Terris, J. E. Stern, D. Rugar, and H. J. Mamin, Phys. Rev. Lett. 63, 2669 (1989)

${ }^{2}$ D. M. Schaadt, E. T. Yu, S. Sankar, and A. E. Berkowitz, Appl. Phys. Lett. 74, 472 (1999).

${ }^{3}$ T. D. Krauss and L. E. Brus, Phys. Rev. Lett. 83, 4840 (1999).

${ }^{4}$ P. A. Rosenthal, E. T. Yu, R. L. Pierson, and P. J. Zampardi, J. Appl. Phys. 87, 1937 (2000).

${ }^{5}$ E. A. Boer, M. L. Brongersma, H. A. Atwater, R. C. Flagan, and L. D. Bell, Appl. Phys. Lett. 79, 791 (2001).

${ }^{6}$ R. Ludeke and E. Cartier, Appl. Phys. Lett. 78, 3998 (2001).

${ }^{7}$ S. Cunningham, I. A. Larkin, and J. H. Davis, Appl. Phys. Lett. 73, 123 (1998).

${ }^{8}$ T. Melin, D. Deresmes, and D. Stievenard, Appl. Phys. Lett. 81, 5054 (2002).

${ }^{9}$ S. Watanabe, K. Hane, T. Ohye, M. Ito, and T. Goto, J. Vac. Sci. Technol. B 11, 1774 (1993).

${ }^{10}$ S. Belaidi, P. Girard, and G. Leveque, J. Appl. Phys. 81, 1023 (1997)

${ }^{11}$ Z. Y. Li, B. Y. Gu, and G. Z. Yang, Phys. Rev. B 57, 9225 (1998).

${ }^{12}$ J. Colchero, A. Gil, and A. M. Baro, Phys. Rev. B 64, 245403 (2001).

${ }^{13}$ S. Gomez-Monivas, L. S. Froufe, R. Carminati, J. J. Greffet, and J. J. Saenz, Nanotechnology 12, 496 (2001).

${ }^{14}$ S. Gomez-Moñivas, L. S. Froufe-Pérez, A. J. Caamaño, and J. J. Sáenz, Appl. Phys. Lett. 79, 4048 (2001).

${ }^{15}$ Y. Martin, C. Williams, and H. K. Wickramasinghe, J. Appl. Phys. 61, 4782 (1987)

${ }^{16}$ T. R. Albrecht, P. Grutter, D. Horne, and D. Rugar, J. Appl. Phys. 69, 668 (1991).

${ }^{17}$ D. W. Abraham, C. Williams, J. Slinkman, and H. K. Wickramasinghe, J. Vac. Sci. Technol. B 9, 703 (1991).

${ }^{18}$ Q. Xu, J. W. P Hsu, J. A. Carlin, R. M. Sieg, J. J. Boeckl, and S. A. Ringel, Appl. Phys. Lett. 75, 2111 (1999).

${ }^{19}$ T. Hochwitz, A. K. Henning, C. Levey, C. Daghlian, and J. Slinkman, J. Vac. Sci. Technol. B 14, 457 (1996). 
${ }^{20}$ E. Tevaarwerk, P. Rugheimer, O. M. Castellini, D. G. Keppel, S. T. Utley, D. E. Savage, M. G. Lagally, and M. A. Eriksson, Appl. Phys. Lett. 80, 4626 (2002).

${ }^{21}$ C. Staii, A. T. Johnson, and N. J. Pinto, Nano Lett. 4, 859 (2004).

${ }^{22}$ M. Bockrath, N. Markovic, A. Shepard, M. Tinkham, L. Gurevich, L. P. Kouwenhoven, M. W. Wu, and L. L. Sohn, Nano Lett. 2, 187 (2002).

${ }^{23}$ FLEXPDE VERSION 3.10.1 (PDE Solutions Inc, Antioch, CA).
${ }^{24}$ To ensure correct gridding, we guide the gridding process around significant topographic features.

${ }^{25}$ F. J. Giessibl, Appl. Phys. Lett. 78, 123 (2001).

${ }^{26}$ The cumulative uncertainty in these equations is $\sim 3 \%$.

${ }^{27} r_{\text {influence }}=0.8 z+4 \mathrm{~nm}$, where $z=200 \mathrm{~nm} / \varepsilon_{\mathrm{SiO}_{2}}+$ tip-sample separation, so $r_{\text {influence }}=60 \mathrm{~nm}$ at a lift scan height of $20 \mathrm{~nm}$. 